\title{
Reanalysis of the high energy cutoff of the 1997 Mkn 501 TeV energy spectrum
}

F. A. Aharonian ${ }^{1}$, A. G. Akhperjanian ${ }^{7}$, J. A. Barrio ${ }^{2,3}$, K. Bernlöhr ${ }^{1}$, O. Bolz ${ }^{1}$, H. Börst ${ }^{5}$, H. Bojahr ${ }^{6}$, J. L. Contreras ${ }^{3}$, J. Cortina ${ }^{2}$, S. Denninghoff ${ }^{2}$, V. Fonseca ${ }^{3}$, J. C. Gonzalez ${ }^{3}$, N. Götting ${ }^{4}$,

G. Heinzelmann ${ }^{4}$, G. Hermann ${ }^{1}$, A. Heusler ${ }^{1}$, W. Hofmann ${ }^{1}$, D. Horns ${ }^{4}$, A. Ibarra ${ }^{3}$, C. Iserlohe ${ }^{6}$, I. Jung ${ }^{1}$,

R. Kankanyan ${ }^{1,7}$, M. Kestel ${ }^{2}$, J. Kettler ${ }^{1}$, A. Kohnle ${ }^{1}$, A. Konopelko ${ }^{1, \S}$, H. Kornmeyer ${ }^{2}$, D. Kranich ${ }^{2}$, H. Krawczynski ${ }^{1}$, H. Lampeitl ${ }^{1}$, E. Lorenz ${ }^{2}$, F. Lucarelli ${ }^{3}$, N. Magnussen ${ }^{6}$, O. Mang ${ }^{5}$, H. Meyer ${ }^{6}$, R. Mirzoyan ${ }^{2}$, A. Moralejo ${ }^{3}$, L. Padilla ${ }^{3}$, M. Panter ${ }^{1}$, R. Plaga ${ }^{2}$, A. Plyasheshnikov $^{1, \S}$, J. Prahl $^{4}$, G. Pühlhofer ${ }^{1}$, W. Rhode ${ }^{6}$, A. Röhring ${ }^{4}$, G. P. Rowell ${ }^{1}$, V. Sahakian ${ }^{7}$, M. Samorski $^{5}$, M. Schilling $^{5}$, F. Schröder ${ }^{6}$, M. Siems ${ }^{5}$, W. Stamm ${ }^{5}$, M. Tluczykont ${ }^{4}$, H. J. Völk ${ }^{1}$, C. Wiedner ${ }^{1}$, and W. Wittek ${ }^{2}$

1 Max Planck Institut für Kernphysik, Postfach 103980, 69029 Heidelberg, Germany

2 Max Planck Institut für Physik, Föhringer Ring 6, 80805 München, Germany

3 Universidad Complutense, Facultad de Ciencias Físicas, Ciudad Universitaria, 28040 Madrid, Spain

${ }^{4}$ Universität Hamburg, II. Institut für Experimentalphysik, Luruper Chaussee 149, 22761 Hamburg, Germany

${ }^{5}$ Universität Kiel, Institut für Experimentelle und Angewandte Physik, Leibnizstraße 15-19, 24118 Kiel, Germany

${ }^{6}$ Universität Wuppertal, Fachbereich Physik, Gaußstr.20, 42097 Wuppertal, Germany

7 Yerevan Physics Institute, Alikhanian Br. 2, 375036 Yerevan, Armenia

$\S$ On leave from Altai State University, Dimitrov Street 66, 656099 Barnaul, Russia

Received 18 August 2000 / Accepted 24 October 2000

\begin{abstract}
Data taken with the HEGRA system of imaging atmospheric Cherenkov telescopes during the 1997 flares of Markarian 501 (Mkn 501) are reanalyzed using an algorithm providing improved energy resolution. A resolution of $10 \%$ to $12 \%$ is obtained by accounting for the variation of the Cherenkov light yield with the height of the shower maximum in the atmosphere. The improved energy resolution is particularly relevant for the study of the high-energy cutoff in the spectrum, which might be caused by interactions with the intergalactic infrared background radiation. The reanalysis presented here confirms the results obtained in the previous analysis, but hints a steeper slope of the spectrum in the region around $20 \mathrm{TeV}$.
\end{abstract}

Key words. galaxies: BL Lacertae objects: individual: Mkn 501 - gamma-rays: observations

\section{Introduction}

The attenuation of extragalactic gamma rays in the $\mathrm{TeV}$ energy range due to interactions with the cosmic infrared background radiation (Nikishov 1962; Gould \& Schréder 1967; Stecker et al. 1992) is under intense discussion (see, e.g., Funk et al. 1998; Stecker \& de Jaeger 1998; Aharonian et al. 1999a; Coppi \& Aharonian 1999; Konopelko et al. 1999a; Stecker 1999; Krawczynski et al. 2000; Vassiliev 2000; and references given therein), in particular since the energy spectra determined for the AGN Mkn 501 (Aharonian et al. 1999a, 1999b, 1999c; Samuelson et al. 1998; Djannati-Atai et al. 1999), obtained during its 1997 flares, show indications of absorption

Send offprint requests to: W. Hofmann, e-mail: Werner.Hofmann@mpi-hd.mpg.de features, particularly evident in the high-energy HEGRA data of Aharonian et al. (1999a), in the following referred to as Paper I. The level of the cosmic infrared background is related to the evolution of galaxies and can serve to distinguish different cosmological models (see, e.g., Primack et al. 1999). However, due to the intense foreground radiation, a reliable direct measurement of the infrared background is a difficult task. Gamma rays in the TeV energy range provide an independent and complementary approach to study the infrared background radiation. Since the $\gamma \gamma \rightarrow \mathrm{e}^{+} \mathrm{e}^{-}$interaction cross section peaks near threshold, the absorption of gamma rays of energy $E$ probes the background level around a wavelength $\lambda / \mu \mathrm{m} \approx \kappa E / \mathrm{TeV}$, where $\kappa$ varies between 1 and 2 depending on the spectral shape of the background radiation. There is almost a one-to-one correspondence 



Fig. 1. a) Attenuation of $\mathrm{TeV}$ gamma rays from $\mathrm{Mkn} 501$ due to interaction with the infrared background radiation, for different assumptions concerning the density of the infrared background. Dotted line: following recent data from Finkbeiner et al. (2000), as shown by the solid line of Fig. 1 in Protheroe \& Meyer (2000). Dashed-dotted line: using the minimal flux consistent with the $1 \sigma$ statistical and systematic error bars of Finkbeiner et al. (2000). Full line: earlier LCFDM model of Primack et al. (1999). All curves use are calculated for the redshift $z \approx 0.03$ of Mkn 501, assuming a Hubble constant of $65 \mathrm{~km} \mathrm{~s}^{-1} \mathrm{Mpc}^{-1}$. b) Spectral energy distribution $E^{2} \mathrm{~d} N / \mathrm{d} E$ of $\mathrm{TeV}$ gamma rays from $\mathrm{Mkn} 501$ at the source, reconstructed by dividing the measured distribution (HEGRA, Paper I, shown as triangles) by the attenuation. Filled circles: using the attenuation indicated by the dotted curve in part (a) of this figure. Open circles: using the attenuation indicated by the dashed-dotted curve, shifting the HEGRA data down in energy by $15 \%$ - the maximal systematic error on the energy scale - and including the systematic error bars of the HEGRA measurement

between the energy-dependent attenuation and the spectral density of the infrared background radiation. Recent analyses by Finkbeiner et al. (2000) of the background level at long wavelengths, at $60 \mu \mathrm{m}$ and $100 \mu \mathrm{m}$, show a significantly higher flux than expected, which results in strong absorption of Mkn 501 gamma rays above $10 \mathrm{TeV}$ (Fig. 1a). While earlier analyses using a lower level of the infrared background (the LCFDM model of Primack et al. 1999, is illustrated in Fig. 1a as one example) found good consistency with the $\mathrm{TeV}$ data (see, e.g., Konopelko et al. 1999a), these new results appear to be in contradiction with measurements using the HEGRA Cherenkov telescopes, which show a gamma-ray flux from Mkn 501 up to at least $16 \mathrm{TeV}$ (Paper I). In order to explain the observed flux in the presence of strong highenergy attenuation, the spectral distribution of gammarays at the source would have to exhibit a rise at high energies (Fig. 1b), inconsistent both with acceleration models and with energetics of the source (see, e.g., Paper I and Coppi \& Aharonian 1999; Guy et al. 2000; Protheroe \& Meyer 2000; Finkbeiner et al. 2000; and references given therein). Exotic solutions of this "gamma-ray crisis" have been proposed, such as violations of Lorentz invariance (Kifune 1999) or photon condensates (Harwit et al. 1999); the latter explanation has been questioned on rather general grounds (Levinson 2000) and seems already to be ruled out by a recent HEGRA analysis (Aharonian et al. 2000).
The TeV gamma-ray data and the infrared background data can be made marginally consistent by fully exploiting the systematic errors of both measurements, i.e., taking the minimal infrared flux values and gamma ray flux values and reducing the HEGRA energy scale by $15 \%$. In this case, a marginally acceptable source spectrum is achieved, see Fig. 1b, in particular given that the highest HEGRA data point is, at the $2 \sigma$ level, consistent with spill-over from the lower energy bins. The bulk of the change in the calculated source spectrum - a factor 100 for the highest data point - can be attributed to the uncertainty in the infrared data; the effect of the HEGRA uncertainties is smaller - about a factor 10 for the highest point.

The interpretation of the experimental results is currently limited by the systematic errors both of the measurement of the infrared background, and of the TeV spectra. One potentially very important factor is the energy resolution in the detection of the $\mathrm{TeV}$ gamma rays. The HEGRA system of Cherenkov telescopes, which provides the highest-energy data, has an energy resolution around 20\% (see Aharonian et al. 1999b; Konopelko et al. 1999b; Paper I). While this resolution is ample for the reconstruction of the usual power-law energy spectra, it may smear out the extremely sharp cutoff features expected for absorption due to infrared background, in particular for high long-wavelength infrared background levels (Fig. 1a). The highest-energy gamma rays detected could simply represent spill-over from lower energies due to the finite energy resolution. Indeed, while Aharonian et al. (Paper I) 
measure photons with reconstructed energies up to $21.5 \mathrm{TeV}$ and beyond, they state that a sharp cutoff at $16 \mathrm{TeV}$ cannot be excluded by the data. It is obviously of considerable interest to improve the energy reconstruction of the system of telescopes, in order to more precisely map a possible cutoff. A scheme to improve the energy resolution of systems of Cherenkov telescopes by roughly a factor 2 has been discussed by Hofmann et al. (2000), making use of the abundant information provided by such systems for each individual gamma-ray air shower. In this note, we present a reanalysis of the HEGRA Mkn 501 spectral data with such improved analysis algorithms.

\section{Determination of gamma ray spectra}

One of the key limiting factors in the energy determination of air showers with imaging Cherenkov telescopes is the fluctuation in the height of the shower maximum in the atmosphere. Deeper showers have a larger light yield both because of the reduced distance to the telescopes and because of the reduction of the threshold for Cherenkov light emission with decreasing height, which allows more particles to contribute to the emission of Cherenkov light. As demonstrated by Hofmann et al. (2000) and by Aharonian et al. (2000), the multiple views of a shower provided by the HEGRA stereoscopic system of Cherenkov telescopes allow to determine the height of the shower maximum with an error of $500 \mathrm{~m}$ to $600 \mathrm{~m}$. Using look-up tables which depend both on the distance to the shower core and the shower height to relate the Cherenkov light yield to the gamma-ray energy, the energy resolution of the HEGRA system can be improved significantly (Hofmann et al. 2000). A further improvement can be reached, if the known location of the gamma-ray source is used as input in the reconstruction of the shower geometry, providing a greatly improved localization of the shower core, and hence a better measurement of the distance of a given telescope to the shower core. Combining all these techniques, an energy resolution around $10 \%$ to $12 \%$ is obtained (Hofmann et al. 2000), implying - for the identical set of events - a factor 2 improvement over more conventional techniques such as used in Aharonian et al. (1999b) and in Paper I.

The new analysis technique was applied to the data collected with the HEGRA telescope system during the extraordinary 1997 flares of Mkn 501. The telescope hardware, the event samples, the event reconstruction and event selection, and the determination of energy spectra are described in Aharonian et al. (1999b), and in Paper I. In particular, relatively loose cuts on the shower direction and in the image shapes are used to select gamma rays from Mkn 501, in order to minimize the efficiency corrections and the systematic errors resulting from these corrections, and events are only used up to core distances of $200 \mathrm{~m}$ from the central telescope. In the current analysis, the new procedure is employed to determine the shower energy on the basis of the measured height of the shower maximum, and the core location is determined given the known source location. We will, in the following, only address the energy range above $3 \mathrm{TeV}$, where the effective detection area of the telescope system is approaching a constant and where threshold effects are negligible. The look-up tables used in the energy determination were optimized for this energy range. At lower energies, errors in the determination of the energy spectra are dominated by the modeling of trigger efficiencies (see Paper I), and no attempt was made to refine the spectra in this range. According to Monte Carlo simulations, the improved energy estimate provides an energy resolution of $10 \%$ to $12 \%$ and has a systematic deviation between the true energy and the average reconstructed energy of less than $\pm 3 \%$, in the range between $3 \mathrm{TeV}$ and $30 \mathrm{TeV}$. Figure 2 compares the performance of this energy reconstruction with the previous technique; the new approach provides both better resolution and reduced non-Gaussian tails.

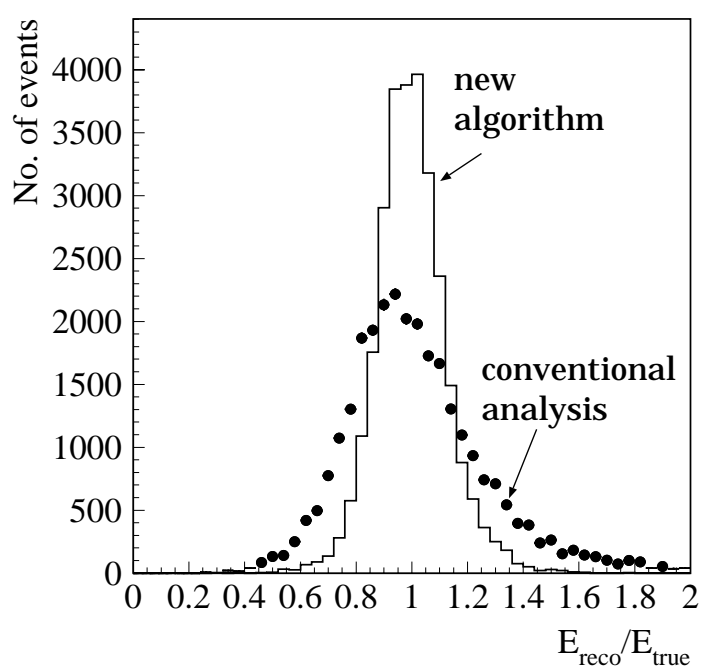

Fig. 2. Ratio of the reconstructed and true gamma-ray energies, as derived from Monte-Carlo simulations at $20^{\circ}$ zenith angle, for energies between $3 \mathrm{TeV}$ and $30 \mathrm{TeV}$. Histogram: new algorithm, points: conventional analysis

\section{Tests of the reconstruction algorithm}

A key issue is of course whether the Monte-Carlo simulation (Konopelko et al. 1999b) - from which the energy look-up tables are derived - is accurate enough to guarantee that the identical energy resolution is obtained for the simulations and the real data. Lacking a monoenergetic test beam, the energy resolution of Cherenkov telescopes cannot be determined directly. Fortunately, however, the information provided by the up to four views of a given shower is highly redundant, and allows a number of cross checks, some of which are briefly summarized in the following. We address in sequence the reconstruction of the shower core, the determination of the shower height, and the energy determination and influence of the atmosphere.

In most of the events in the range above $3 \mathrm{TeV}$, all four telescopes trigger and provide images; the mean number 
of telescopes used in the reconstruction is 3.7. The precision in the location of the shower core can be tested by reconstructing, in four-telescope events, the same shower independently by two stereo subsystems of two telescopes each. The rms difference in the $x$ or $y$ coordinates of the core, comparing two telescopes with the other two, is about $3.1 \mathrm{~m}$ for small core distances, and grows to $5.6 \mathrm{~m}$ for distances of $100 \mathrm{~m}$. These differences are about $30 \%$ larger than predicted by the simulation, indicating remaining alignment errors. The same simulation predicts an error in the core coordinates of less than $2 \mathrm{~m}$ rms over the full distance range, when combining all telescopes. Scaled up by $30 \%$, this error is, however, still safely below values which could affect the energy determination.

For the energy determination, it is crucial that the simulation provides a proper modeling of the atmosphere, and in particular that the average height of the shower maximum is reproduced. Otherwise, the energy correction based on the height of the shower maximum might introduce a serious bias. Figure 3 shows, as a function of zenith angle, the measured shower height, compared with simulations. In order not to introduce a bias by cuts on the - potentially biased - shower energy, events were selected by requiring two telescopes with at least 100 photoelectrons. Data and simulation agree to better than $2 \%$. Simulations show that even a $5 \%$ mismatch in the average shower height has an almost negligible effect on the energy resolution, and introduces a bias in the overall energy scale of less than 3-4\%. Also the widths of the distributions of shower heights agree within statistical errors between data and simulation; at $20^{\circ}$ zenith angle, e.g., the measured (Gaussian) width is $0.82 \pm 0.02 \mathrm{~km}$, compared to $0.80 \pm 0.01 \mathrm{~km}$ in the simulation. We note that due to the selection condition used for the data of Fig. 3, the energy of gamma rays increases with increasing zenith angle. The zenith-angle dependence of the average shower height is caused by a combination of two effects: at larger angles (and fixed energy), the shower maximum moves up since the amount of radiation lengths traversed at a given height increases like $1 / \cos \left(\theta_{\text {zenith }}\right)$; however, showers at larger zenith angles require a higher energy to provide two images above 100 photoelectrons and therefore penetrate deeper.

Checks of the energy resolution use the feature that for a given shower and a core location determined on the basis of the orientation of the images, the light yield observed in each individual telescope can be converted into an energy estimate. For the final energy value these estimates are averaged, but the consistency of the up to four energy values for each shower provides stringent tests of the quality of the reconstruction procedure.

A crucial input in the energy reconstruction is the dependence of the measured light yield on the distance to the shower core. This input can be checked by comparing the energy estimates of two telescopes located at different distances from the shower core. We find that the (averaged) ratio of the energy estimates provided by two telescopes shows a systematic variation with the two core distances

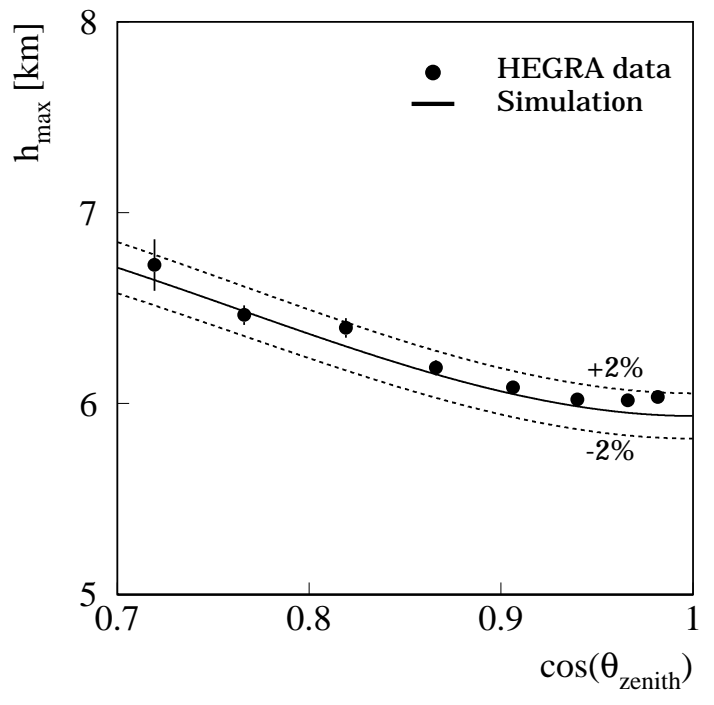

Fig. 3. Points: average reconstructed shower height for gamma-ray showers as a function of zenith angle, for events with at least two images above 100 photoelectrons. Full line: Monte Carlo simulations. Dashed lines: $\pm 2 \%$ range around simulation results

(in the range between 0 and $200 \mathrm{~m}$ ), at a level of $4.2 \%$ rms. A $4.2 \%$ systematic variation should not spoil the $10 \%$ energy resolution; furthermore, the effect is fully reproduced by the simulations, which show, under the same conditions, a $4.4 \%$ rms variation.

The previous test checks if the simulations reproduce the variation of the mean light yield with core distance. An equally important test is to see if the fluctuations are properly described. Using four-telescope events, the median rms spread of the four individual energy estimates was determined to $12.3 \pm 0.3 \%$, slightly below the Monte Carlo value of $13.1 \%$. The conventional energy reconstruction shows a $19.5 \pm 0.5 \%$ median rms spread between telescopes, compared to the simulation value of $19.2 \%$; shower height fluctuations result in strong correlations between the individual telescopes, and govern the energy resolution.

Finally, one has to worry about varying atmospheric conditions, which might introduce time-dependent variations in the light yield. Since such effects will most likely influence all telescopes in more or less the identical manner, they would not show up in inter-telescope comparisons. One can distinguish two effects: long-term seasonal variations and short-term variations. The influence of seasonal variations on the Cherenkov light yield is discussed by Bernlöhr (2000). Atmospheric density profiles and temperature profiles on the Canary Islands are continuously monitored by radiosondes; on the basis of these data (Cuevas 1997) and of the simulations of Bernlöhr (2000) we conclude that the seasonal variation of the Cherenkov light yield should be less than $2 \%$ rms for the Mkn 501 data set. More critical are short-term variations in atmospheric transmission. As discussed in Paper I, the data set used here is selected to exclude nights with excessive 
attenuation. To monitor the attenuation, the trigger rate of the telescope system is used; the rate varies roughly like the 1.6th power of the energy threshold and variations in the transmission cause corresponding rate variations, amplified by a factor 1.6. For the data set after selection, the mean trigger rate (at a given zenith angle) varies day-byday by $4 \% \mathrm{rms}$ for the first period of data, and by $6 \% \mathrm{rms}$ for periods 2 and 3 (Aharonian et al. 1999b; by mistake, the paper quotes $4 \% \mathrm{FWHM}$ instead of the correct value of $4 \% \mathrm{rms}$ ). These rate fluctuations translate into fluctuations in the light yield - and hence of the energy scale - of $3 \%$ to $4 \% \mathrm{rms}$, sufficiently small compared to the resolution of $10 \%$ to $12 \%$ rms.

On the basis of these and other tests, we believe that in the data there are no systematic effects or additional fluctuations, which would significantly worsen the energy resolution compared to the resolution predicted by the simulations.

Concerning the absolute energy scale, the new analysis is exactly like the previous analyses susceptible to imperfections in the modeling of the atmosphere, of the transmission of the telescope optical systems, and of the photomultiplier and electronics gains. Therefore, the $15 \%$ uncertainty on the energy scale discussed in Paper I also applies here. The same is true for systematic errors at high energies due to photomultiplier nonlinearities or electronics saturation.

\section{Results and interpretation}

Figure 4 shows the spectral energy distribution obtained with the new energy reconstruction, together with the previous results of Paper I. The new data points are listed in Table 1. Except for the highest point in the previous data set, at $E=21.45 \mathrm{TeV}$, the two analyses are in very good agreement. In Paper I, the influence of the energy resolution was addressed by studying a model, where the source spectrum shows a power law with an index around 2, an exponential cutoff, and in addition a sharp cutoff at an energy $E_{\text {cut }}$. At the $2 \sigma$ level, a hard cutoff below $16 \mathrm{TeV}$ was excluded in Paper I; this result implies that at the $2 \sigma$ level, in particular the data point at $21.45 \mathrm{TeV}$ is consistent with spill-over from lower energies. In the new analysis, no significant signal is observed in this energy range, and upper limits are below the value of the previous point. We conclude therefore that at these very highest energies, the spectrum appears indeed steeper than indicated by the data of Paper I, but consistent within the systematics quoted in Paper I. While this change goes in the expected direction, a statistical fluctuation at the $2-3 \sigma$ level cannot be excluded as the origin of the change, due to the low statistics for this data range in either analysis. In the range up to the data point at $17.08 \mathrm{TeV}$ of Paper I, there is almost no change observed due to the improved energy resolution. Combining the previous data below $3 \mathrm{TeV}$ with the new data points above $3 \mathrm{TeV}$, a good fit to the data

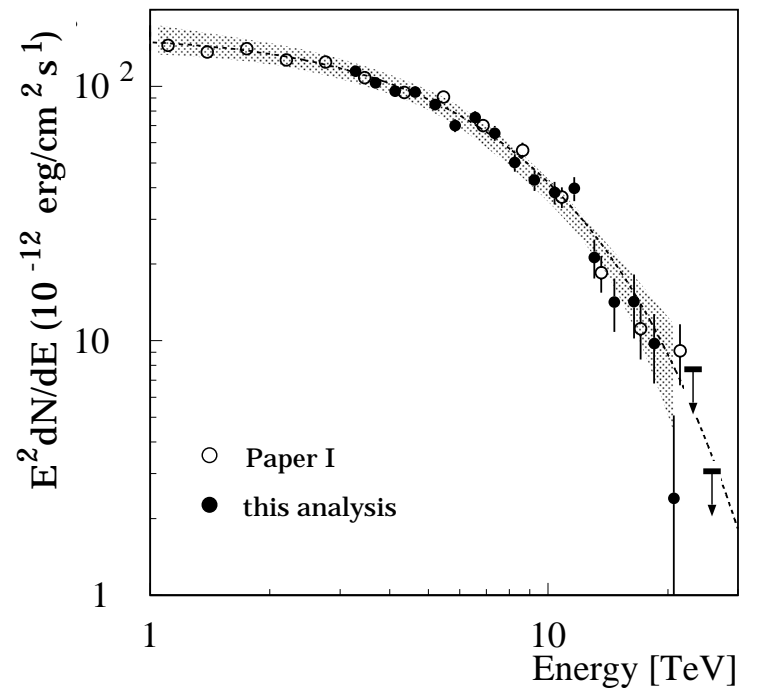

Fig. 4. Spectral energy distribution $E^{2} \mathrm{~d} N / \mathrm{d} E$. Full symbols and upper limits: new analysis with improved energy resolution; open symbols: conventional analysis, see Paper I. The shaded regions indicate systematic errors. In addition, there is a $15 \%$ uncertainty on the absolute energy scale. The dashed line shows the fit $E^{-1.92} \exp \left(-E / E_{\mathrm{o}}\right)$ to the data of Paper I, with $E_{\mathrm{o}}=2.6 \mathrm{TeV}$

Table 1. Spectral energy distribution $E^{2} \mathrm{~d} N / \mathrm{d} E$ in units of $10^{-12} \mathrm{erg} \mathrm{cm}^{-2} \mathrm{~s}^{-1}$. See text for systematic errors

\begin{tabular}{|r|r|r|}
\hline$E[\mathrm{TeV}]$ & $E^{2} \mathrm{~d} N / \mathrm{d} E$ & stat. error \\
\hline 3.28 & 114.7 & 4.6 \\
3.68 & 103.4 & 4.3 \\
4.13 & 95.8 & 4.3 \\
4.63 & 94.8 & 4.4 \\
5.20 & 84.8 & 4.3 \\
5.83 & 70.1 & 4.1 \\
6.54 & 75.4 & 4.5 \\
7.34 & 65.4 & 4.3 \\
8.24 & 50.0 & 4.0 \\
9.25 & 42.8 & 3.9 \\
10.38 & 38.2 & 3.9 \\
11.64 & 39.6 & 4.2 \\
13.06 & 21.2 & 3.7 \\
14.66 & 14.1 & 3.3 \\
16.45 & 14.2 & 4.0 \\
18.45 & 9.7 & 2.9 \\
20.71 & 2.4 & 2.6 \\
\hline
\end{tabular}

is achieved with the parametrization

$\mathrm{d} N / \mathrm{d} E \sim E^{-2.03 \pm 0.03} \exp \left(-\left(E / E_{\mathrm{o}}\right)^{1.31 \pm 0.11}\right)$

with $E_{\mathrm{o}}=8.21 \pm 0.63 \mathrm{TeV}$. The fit indicates that indeed a super-exponential cutoff is favored. We note - as already mentioned in Paper $\mathrm{I}$ - that the fit parameters are strongly correlated and cannot be varied independently 
within their errors. Errors quoted for the fit parameters are purely statistical.

Carrying out a similar analysis with a hard cutoff for the new results, a cutoff below $16.5 \mathrm{TeV}$ can be excluded.

In summary, the conclusions concerning the level of the infrared background allowed by the HEGRA Mkn 501 energy spectra remain unchanged: taken at face value, the HEGRA data and the infrared flux levels of Finkbeiner et al. (2000) are inconsistent; making full use of the systematic errors allowed for the HEGRA data and in particular for the infrared flux determinations, the two data sets can be brought to a marginal consistency, without a clear need to invoke exotic explanations.

Acknowledgements. The support of the HEGRA experiment by the German Ministry for Research and Technology BMBF and by the Spanish Research Council CYCIT is acknowledged. We are grateful to the Instituto de Astrofísica de Canarias for the use of the site and for providing excellent working conditions. We gratefully acknowledge the technical support staff of Heidelberg, Kiel, Munich, and Yerevan. GPR acknowledges receipt of a Humboldt Foundation postdoctoral fellowship.

\section{References}

Aharonian, F. A., Akhperjanian, A. G., Barrio, J. A., et al. 1999a, A\&A, 349, 11

Aharonian, F. A., Akhperjanian, A. G., Barrio, J. A., et al. 1999b, A\&A, 342, 69

Aharonian, F. A., Akhperjanian, A. G., Barrio, J. A., et al. 1999c, A\&A, 349, 29

Aharonian, F. A., Akhperjanian, A. G., Barrio, J. A., et al. 2000, submitted to ApJ [astro-ph/0006092]

Bernlöhr, K. 2000, Astropart. Phys., 12, 255
Cuevas, E. 1997, Izana Global Atmospheric Watch (GAW) Observatory, private communication

Coppi, P. S., \& Aharonian, F. A. 1999, Astroparticle Phys., 11, 35; ApJ, 521, L33

Djannati-Atai, A., Piron, F., Barrau, A., et al. 1999, A\&A, 350,17

Finkbeiner, D. P., Davis, M., \& Schlegel, D. J. 2000 [astro-ph/0004175]

Funk, B., Magnussen, N., Meyer, H., et al. 1998, Astropart. Phys., 9, 97

Gould, J., \& Schréder, G. 1967, PR, 155, 1404

Guy, J., Renault, C., \& Aharonian, F. A., et al. 2000, A\&A, 359, 419

Harwit, M., Protheroe, R. J., \& Biermann, P. L. 1999, ApJ, 524, L91

Hofmann, W., Lampeitl, H., Konopelko, A., \& Krawczynski, H. 2000, Astropart. Phys., 12, 207

Kifune, T. 1999, ApJ, 518, L21

Konopelko, A., Kirk, J. G., Stecker, F. W., Mastichiadis, A. 1999a, ApJ, 518, L13

Konopelko, A., Hemberger, M., Aharonian, F., et al. 1999b, Astropart. Phys., 10, 275

Krawczynski, H., Coppi, P. S., Maccarone T., \& Aharonian, F. A. 2000, A\&A, 353, 97

Levinson, A. 2000 [astro-ph/0007109]

Nikishov, A. I. 1962, Sov. Phys. JTEP, 14, 393

Primack, J. R., Bullock, J. S., Somerville, R. S., \& MacMinn, D. 1999, Astropart. Phys., 11, 93

Protheroe, R. J., \& Meyer, H. 2000 [astro-ph/0005349]

Samuelson, F. W., Biller, S. D., Bond, I. H., et al. 1998, ApJ, 501, L17

Stecker, F. W. 1999, Astropart. Phys., 11, 83

Stecker, F. W., \& de Jager, O. C. 1998, A\&A, 334, L85

Stecker, F. W., De Jager, O. C., \& Salomon, M. H. 1992, ApJ, 390, L49

Vassiliev, V. V. 2000, Astropart. Phys., 12, 217 